\title{
Capacitive properties of zinc oxide thin films by radiofrequency magnetron sputtering
}

\author{
Roger Ondo-Ndong ${ }^{1} \cdot$ Hans Essone-Obame ${ }^{1} \cdot$ Zita Hermance Moussambi $^{1} \cdot$ Nathaniel Koumba $^{1}$
}

Received: 3 May 2018 / Accepted: 8 November 2018 / Published online: 17 November 2018

(c) The Author(s) 2018

\begin{abstract}
The study focused on the production of zinc oxide $(\mathrm{ZnO})$ thin films as a dielectric material for use in metal-insulator-semiconductor capacitors. The objective of this study has demonstrated the frequency dependence of conductivity and capacity. Zinc oxide $(\mathrm{ZnO})$ was thin films deposited by a silicon wavelength sputteringlmagnetron cathode sputtering. The capacitive properties of $\mathrm{ZnO}$ zinc oxide were studied at room temperature. The frequency dependence of the conductivity, capacitance and the measured current-voltage $(I-V)$ characteristics of $\mathrm{ZnO}$ zinc oxide thin films were studied in the frequency range from $5 \mathrm{kHz}$ to $13 \mathrm{MHz}$. It is shown that the conductivity is total. Indeed, the measurement of the conductivity in alternating regime obeys the Arrhenius equation. In addition, the measured $I-V$ characteristics of the structures studied at $10 \mathrm{kHz}$ and $10 \mathrm{MHz}$ clearly revealed areas of accumulation, depletion and inversion in the plots. It has been observed that AC conductivity and capacity in the $\mathrm{ZnO}$ thin films $(\mathrm{ZnO})$ are frequency dependent. This dependence indicates that the conduction is thermally activated and maintains the correlated barrier of the charge carrier on the localized states as a function of the experimental data. The FBAR (expand) devices with the $\mathrm{ZnO}$ films exhibited a pronounced resonance peak centered at $537 \mathrm{MHz}$ with a $k^{2}$ coupling coefficient of 7\%. It found therefore that the impedance matching of the FBAR could be easily achieved simply by controlling the resonance the resonator.
\end{abstract}

Keywords Zinc oxide $\cdot$ Thin films $\cdot$ Sputtering $\cdot$ Current-voltage study $\cdot$ Capacity properties $\cdot$ FBAR device

\section{Introduction}

Currently, zinc oxide as a dielectric material is the subject of particular attention in the development of micro- and nanotechnologies. Indeed, the determination of its electrical properties in metal-insulator-semiconductor (MIS) capacitors as a dielectric material is much studied $[1,2]$. Indeed, AC conductivity measurements provide information about the interior of the insulation or semiconductor which is a region of relatively low conductivity, even when the conduction process is limited to the electrode [3, 4]. The identification of the conduction mechanism depends on both frequency and temperature dependence of electrical conductivity, and Mahmood et al. [5] studied the electrical properties of $\mathrm{ZnO}$ thin films by RF sputtering. It has been found

Roger Ondo-Ndong

roger.ondo_ndong@yahoo.fr

1 Laboratoire Pluridisciplinaire des Sciences (LAPLUS), Ecole Normale Supérieure, Libreville, BP17009, Libreville, Gabon that the electrical conductivity has been thermally activated and dominated by the correlated barrier at low temperature [6].

Indeed, $\mathrm{ZnO}$ has the potential for many applications such as gas sensors. [7-9]. This material is also interesting because of its simple and economical photocatalytic activity. Wide-band-gap semiconductor, $\mathrm{ZnO}$ exhibits interesting optoelectronic properties and spintronic applications [10, 11] because of its direct band-gap and large exciton binding energy ( $60 \mathrm{meV}$ at room temperature) that can be used for UV-blue lasers and diodes [10, 12]. UV and visible band emission provides useful information about the electronic properties of $\mathrm{ZnO}$. The UV emissions are usually attributed to the free excitons and donor bound excitons, whereas deep level emissions usually arise from intrinsic defects in $\mathrm{ZnO}$. The zinc oxide usually shows $n$-type conductivity because of intrinsic defects such as oxygen vacancies and zinc interstitials. Through these mechanisms, $\mathrm{ZnO}$ acts as an excellent antibacterial agent [13]. $\mathrm{ZnO}$ materials have been synthesized through numerous methods, which include chemical precipitation, sol-gel, pulsed laser deposition technique, 
microwave radiation and hydrothermal methods [14]. The doping of $\mathrm{ZnO}$ materials with different types of metallic ions $[11,15,16]$ enhances its optical, magnetic and conducting properties. This modified $\mathrm{ZnO}$ is used as a base material in photocatalysts, dosimeters and biological systems [12]. Furthermore, doping with rare earth elements provides many interesting $\mathrm{ZnO}$ properties, including lanthanum-doped $\mathrm{ZnO}$ with excellent gas sensitivity and photocatalytic activity [17]. Functional dual TMs doping into $\mathrm{ZnO}$ nanostructures could influence the charge carrier recombination rate, optical, structural and interfacial electron-transport rate performance [15].

Also, the determination of the electrical properties of $\mathrm{ZnO}$ as a dielectric material in metal-insulator-semiconductor capacitors is more than necessary in the development of nanotechnology devices.

In the literature, the authors have shown that certain properties are determined by the orientation of the crystallites on the substrate. That is, the FWHM width of the $\mathrm{ZnO}$ peak along the orientation (002) of the X-ray inclination curve should be less than 0.58 to achieve an effective electromechanical coupling $[18,19]$. Other studies have shown the frequency dependence of the electrical properties of $\mathrm{ZnO}$ sandwich structures [20]. The $\sigma_{\mathrm{AC}}$ test based on the electrical properties of the $\mathrm{ZnO}$ thin films showed that in the frequency range of $10 \mathrm{kHz}$ to $10 \mathrm{MHz}$, electrical conduction is performed by tunneling between the quantum wells. The resistivity of the dielectric material being high, the electrical conductivity of the material can only be achieved by transporting the blasting charge between the particles. In this work, the characteristics of metal-insulator-semiconductor (MIS) capacitors with zinc oxide ( $\mathrm{ZnO}$ ) as a dielectric material were studied. $\mathrm{ZnO}$ thin film was analyzed by $\mathrm{X}$-ray diffraction, and the electromechanical coupling coefficient $k^{2}$ was measured with network analyzer.

\section{Experimental}

The RF magnetron sputtering was used to deposit the thin films of $\mathrm{ZnO}$. Indeed, a target of zinc (99.99\%) with a diameter of $51 \mathrm{~mm}$ and $6 \mathrm{~mm}$ of thickness allowed us to make these deposits. The silicon substrate used is of the $p$-type with orientation (100). The deposits were carefully cleaned with organic products. The magnetron sputtering is carried out at a gas/argon mixture atmosphere. A power at frequency of $13.56 \mathrm{MHz}$ is supplied to the system. The RF power was about $50 \mathrm{~W}$. The flow rates of argon and oxygen were monitored using a flowmeter (ASM, AF 2600). The spraying pressure was maintained at $3.35 \times 10^{-3}$ Torr controlled by a Pirani gauge. Deposition rates covered the range of $0.35-0.53 \mu \mathrm{m} / \mathrm{h}$. All films were annealed in an atmosphere of helium at $650{ }^{\circ} \mathrm{C}$ for $15 \mathrm{~min}$. Indeed, we found that the titanium layer in a $\mathrm{Pt} / \mathrm{Ti} / \mathrm{Si}$ structure annealed at $650{ }^{\circ} \mathrm{C}$ for 15 min under an oxygen flow was completely oxidized, while under helium and under the same conditions, the structure exhibited unoxidized titanium layer.

We have previously deposited Ti/Pt as thin inking films on the silicon substrate to facilitate adhesion of the $\mathrm{ZnO}$ films. It has been found that a substrate temperature of $100{ }^{\circ} \mathrm{C}$, target/substrate distance of about $70 \mathrm{~mm}$, very low gas pressures of $3.35 \times 10^{-3}$ Torr in a mixed argon gas atmosphere and for an oxygen content of $20 \%$, giving to $\mathrm{ZnO}$ thin films a good homogeneity and high crystallinity. The thickness measurements of the samples were taken using a DEKTAK_ST high-resolution profilometer. We measured 2.8-3 $\mu \mathrm{m} \mathrm{ZnO}$ thin films on samples. We confirmed these thicknesses by the determination of the optical properties. Indeed, the optical properties are a function of the thickness. To do this, we consider that the two envelopes of the measured optical transmittance form a nonlinear system consisting of continuous functions that can be solved by iteration. These continuous functions are the refractive index and the absorption percentage. After deposition of the zinc oxide, silver dot electrodes were evaporated on the $\mathrm{Pt} / \mathrm{ZnO} / \mathrm{Ti} / \mathrm{Pt} / \mathrm{Si}$ sample using an electron gun evaporation system to render the structure metal-insulator-semiconductor useful for electrical measurements. These were carried out in a vacuum chamber evacuated at about $10^{-3}$ Torr. Measurements of frequency and temperature dependencies of conductivity and capacity were taken using a laboratory configuration for the AC-based electrical test properties. The samples obtained were tested using an alternating current in the frequency range from $5 \mathrm{~Hz}$ to $13 \mathrm{MHz}$ (using a capacitance bridge technique) using measurement temperatures of $\mathrm{Tp}=30-300{ }^{\circ} \mathrm{C}$, with a Hewlett-Packard LF 4192 A impedance analyzer between silver points and $\mathrm{Pt}$ electrode bottom. The temperature was measured with a Doric thermometer (Trendicator $400 \mathrm{~K} /{ }^{\circ} \mathrm{C}$ ). To appreciate the nature of our piezoelectric $\mathrm{ZnO}$ layers, we determined the coupling coefficient of the complete structure based on frequencies for which the conductance is maximum and the minimum susceptance using a network analyzer (HP 8752 A Network Analyzer) whose pass-band ranges from $300 \mathrm{kHz}$ to $1.3 \mathrm{GHz}$. This type of device is one of the instruments to define with precision the characteristics of an electric circuit (impedance measurement, electrical admittance, reflection coefficient, etc.).

\section{Conductivity measurements}

A different mechanism has been proposed to explain the conductivity $\sigma(\omega)$ in chalcogenide glasses [21-23], scandium oxide and glasses [22], zinc oxide [24], ceramic [25] and cobalt ferrite [26]. This mechanism is based on the classic 
jump of charge carriers between the states located above a potential barrier. This process usually involves phonons, due to the height of the barrier. Consider $W_{\mathrm{M}}$ as the energy difference between the ground state of the potential and the ionized (extended) state distant from $R$. Then, $W$ the potential barrier height of the coulombian type is given by the relationship [22]:

$W=W_{\mathrm{M}}-\frac{4 \mathrm{e}^{2}}{\pi \varepsilon R}$

where $e$ is the effective dielectric constant.

In the following, only the cases where the jumps between very close sites are the most important were considered. Assuming random spatial distribution of the sites, Pike obtained the expression:

$\sigma(\omega)=\frac{\varepsilon N N_{\mathrm{p}}}{3 \pi^{3}}\left(\frac{\mathrm{e}^{2}}{\varepsilon W_{\mathrm{M}}}\right)^{6} \frac{\omega^{s}}{\tau^{1-s}}$

with

$s=1-\frac{6 k T}{W_{\mathrm{M}}}$

where $s$ is temperature and frequency dependent. Long [27] has discussed the case where an appreciable overlap of the polaron hopping distortion could occur (OLPT), thereby reducing the value of the polaron hopping energy.

According to Elliott, we can show that Eq. (2) can be rewritten as [28]:

$\sigma(\omega)=a \omega\left(\frac{1}{\omega \tau}\right)^{6 k T / W_{\mathrm{M}}}$

where $\tau$ is minimum relaxation time.

This equation accounts for the variation of $\sigma(\omega)$ with the temperature $T$, then:

$\frac{\mathrm{d} \ln \sigma(\omega)}{\mathrm{d} \ln \left(\frac{1}{T}\right)}=\frac{6 k T^{2}}{W_{\mathrm{M}}} \ln (\omega \tau)$

The absolute value of this slope decreases as the temperature decreases. This slope also depends on the frequency. For a given temperature, this slope increases when $\omega$ decreases.

If we take

$\beta=1-s$

as a function of temperature, we obtain a straight line that passes through the origin. From this slope, we deduce $W_{\mathrm{M}}$. The value found for $W_{\mathrm{M}}$ is to be compared with the activation energies necessary for the hopping of polaron. Holstein showed that at high temperature, the hopping of a small polaron is essentially a transition over the barrier [25], so the Pike model can be applied to the polaron hopping.

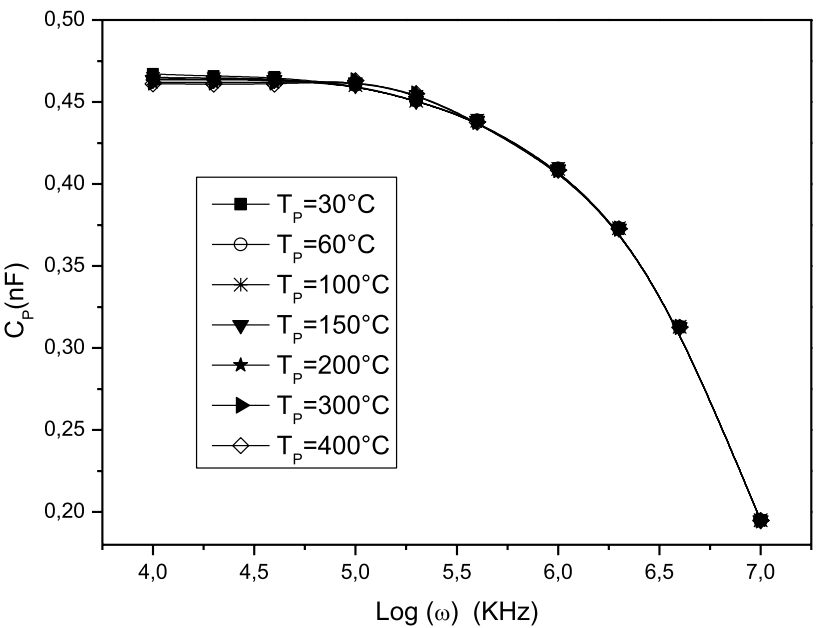

Fig. 1 Capacitance versus frequency dependence

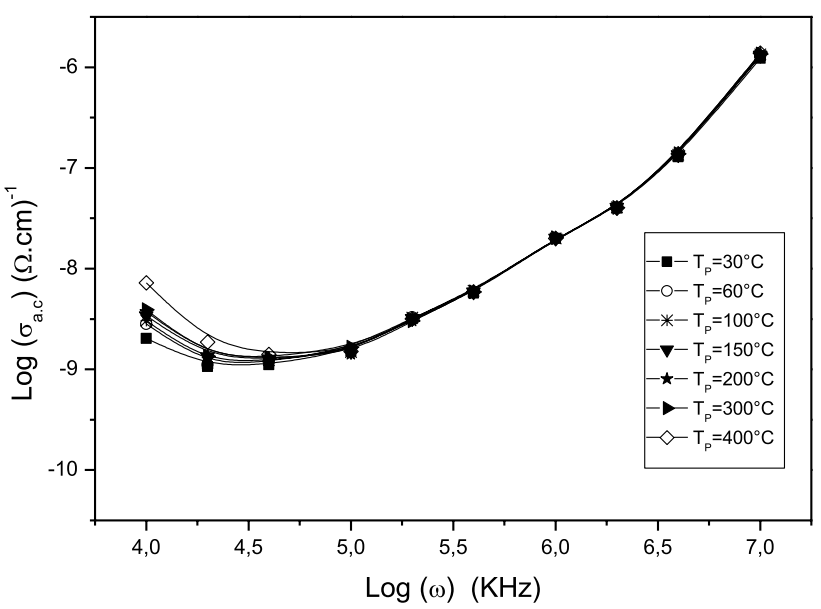

Fig. 2 Dependence of the total AC conductivity in $\mathrm{ZnO}$ films deposited on silicon substrate at different temperature

The frequency dependence of the conductivity, for the sample grown at $100{ }^{\circ} \mathrm{C}$ and for different temperatures, is shown in Fig. 1. The frequency dependence of the capacitance is shown in Fig. 2.

The linear scale was chosen for their presentation in order to determine the mean values of the relaxation times of the permittivity and of the conductivity. Its shown that at the angular frequency:

$\omega=2 \pi f=1 / \tau$

and

$\varepsilon_{\mathrm{r}}=C_{\mathrm{P}} / C_{0}$,

the dielectric permittivity value decreases twice while the conductivity double its value at higher frequencies. This means that the analysis of the changes in the frequency dependencies is shown in Figs. 1 and 2 which makes it easy 
to determine the average values of the time constants for the changes in the average capacitance and the conductivity. The mean values of the relaxation time of the permittivity and of the conductivity can be determined using, respectively, the graphs in Figs. 1 and 2 which show that for capacitance and conductivity, the average value of the relaxation time is about $8 \times 10^{-8} \mathrm{~s}$. This means that in the $\mathrm{ZnO}$ tested there is a correlation between the increase in conductivity and the decrease in capacity in their variations.

In order to identify the conduction mechanism in these thin films, the frequency exponent $s$ is plotted as a function of temperature and deposition frequency a decrease in $s$ with an increasing frequency as shown in Fig. 3. On the basis of the discussion, the variation of $s$ with the frequency is compatible with the overlap polaron tunnel model (OLPT) for a long polaron ray model developed by Long [27]. The variation of the exponent $s$ with the temperature is illustrated in Fig. 4 for different frequency values. It is observed that $s$ decreases at the temperature of $150{ }^{\circ} \mathrm{C}$, and beyond this temperature, $s$ increases. In addition, the value of $s$ was higher when measured at a lower frequency. The low-temperature results were consistent with calculations based on a model proposed by Holstein (2918) to explain the AC conductivity of amorphous materials. Indeed, it showed that at high temperature, the jump of a small polaron is essentially a transition on the barrier [28, 29], so that the Pike model can be applied to the polaron jump. It would therefore seem that the decrease in $s$ with frequency, especially at lower temperatures, is related to a gradual transition between different jump regimes. Therefore, the correlated barrier $(\mathrm{CBH})$ and large polaron ray overlap (OLP) models are suitable for interpreting $\mathrm{ZnO}$ data deposited at $100{ }^{\circ} \mathrm{C}$.

To determine activation energy values, Arrhenius plots were plotted for capacity (Fig. 5) and conductivity (Fig. 6) for

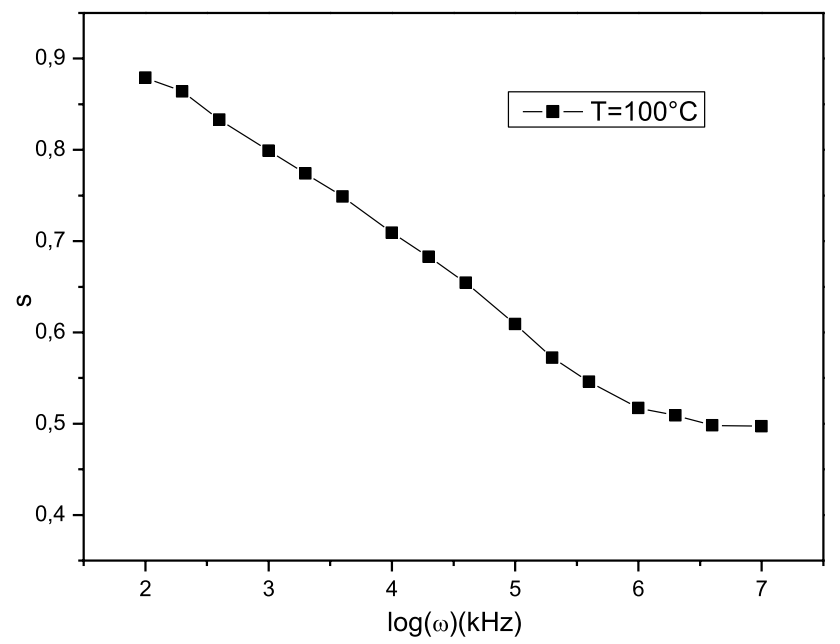

Fig. 3 Frequency dependence of the frequency parameter $s$ at substrate temperatures of $100{ }^{\circ} \mathrm{C}$

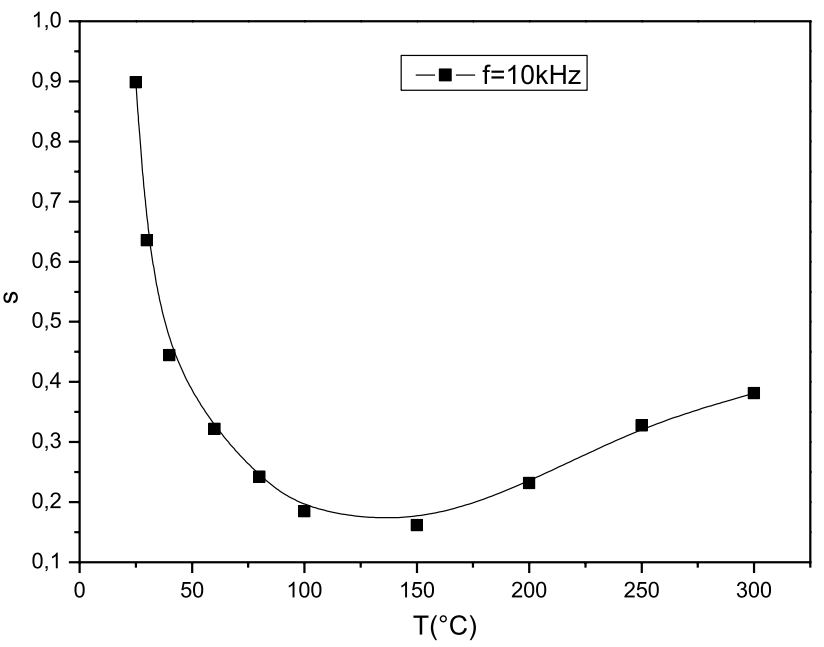

Fig. 4 Temperature dependence of the frequency parameter $s$ at frequency of $10 \mathrm{kHz}$

measuring frequencies of $10 \mathrm{kHz}$ and $10 \mathrm{MHz}$. The activation energy values determined in relation to these plots in the case of capacity are $\Delta E_{C 1} \approx 1.82 \mathrm{meV}$ for the frequency of $10 \mathrm{kHz}$ and $\Delta E_{C 2} \approx 0.43 \mathrm{meV}$ for the frequency of $10 \mathrm{MHz}$. In the case of conductivity for the frequency of $10 \mathrm{kHz}$, the activation value of the energy is $\Delta E_{\sigma 1} \approx 24.4 \mathrm{meV}$, while for the frequency of $10 \mathrm{MHz}$, the value is $\Delta E_{\sigma 2} \approx 2.27 \mathrm{meV}$.

The electrical conduction of thin films ZnO MIS transistors allowed us to measure the capacitance and the dielectric constant using the following formula:

$C_{\mathrm{p}}=\frac{\varepsilon_{\mathrm{r}} \varepsilon_{0} A}{d}$,

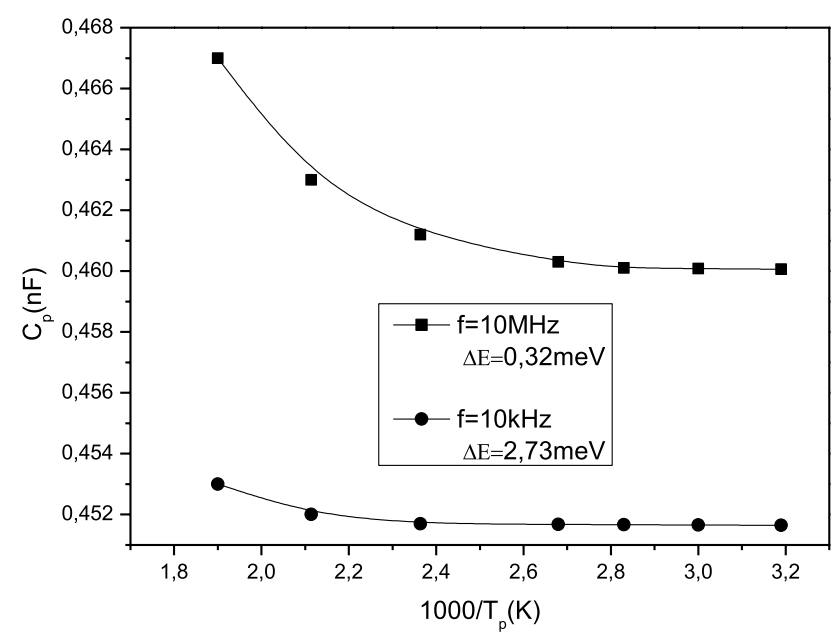

Fig. 5 Arrhenius plot for capacitance for the frequency values of $10 \mathrm{kHz}$ and $10 \mathrm{MHz}$ 


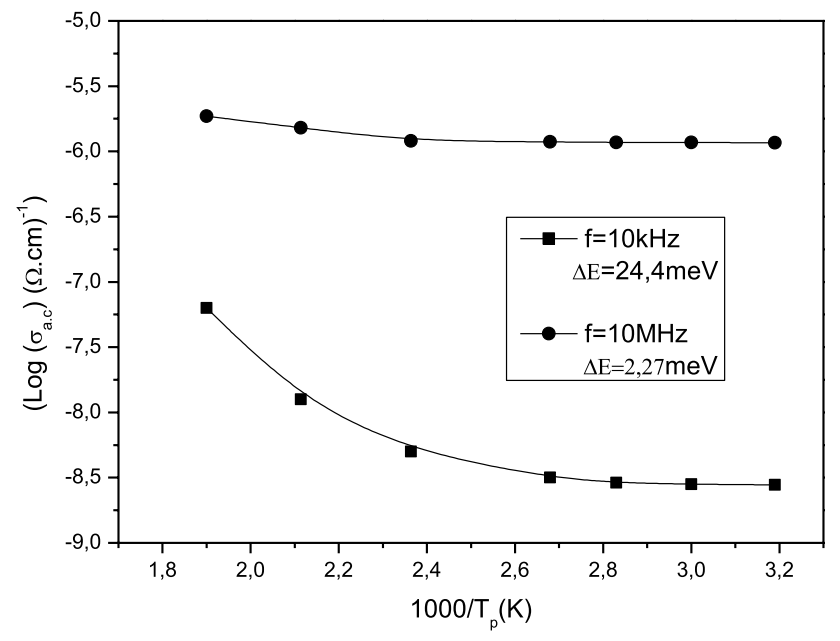

Fig. 6 Arrhenius plot for conductivity for the frequency values of $10 \mathrm{kHz}$ and $10 \mathrm{MHz}$

where $C_{\mathrm{p}}$ is the capacitance, $\varepsilon_{\mathrm{r}}$ is the relative dielectric constant, $\varepsilon_{0}$ is the dielectric constant of void, $A$ is the active area of the capacitor and $d$ is the thickness of a dielectric layer.

The dissipation factor is given by the formula:

$\tan \phi=\frac{\sigma}{\varepsilon_{\mathrm{r}} \varepsilon_{0} \omega}$,

where $\tan \phi$ is the dissipation factor, $\sigma$ is the conductivity and $\omega$ is the pulsation. This gave an average of 8.5 for the value of the permittivity and a very low dissipation factor of $10^{-4}$ for the sample deposited at $100{ }^{\circ} \mathrm{C}$ compared to the samples deposited below and above $100{ }^{\circ} \mathrm{C}$, where the permittivity values are smaller. Similarly, for the dissipation factor, we observe an increase in their values. These capacity data confirm that the $\mathrm{ZnO} / \mathrm{Si}$ interface does not form a Schottky barrier and that the measurements of the capacity dependence on the film thickness indicate that the relative permittivity of the films is about 8.5 . The higher temperature results can be explained by assuming an electron concentration at room temperature and a space-limited conductivity dominated by traps exponentially distributed with energy $E$ under the edge of the web of conduction according to the relation

$N=N_{0} \exp \left(-\frac{\Delta E}{k_{\mathrm{B}} T}\right)$,

where $k_{\mathrm{B}}$ is Boltzmann constant [5]. It results that at low frequency, the electrical capacity of a material containing a potential well is not dependent on the frequency. When the angular frequency $\omega$ approaches $1 / \tau$, the value of the electrical capacitance begins to decrease and tends toward a characteristic value of a material which does not contain any potential wells. The relaxation time in this case gives us relation which is exponentially dependent on the temperature $[30,31]$ :

$\tau(T)=\tau_{0} \cdot \exp \left(\frac{\Delta E}{k_{\mathrm{B}} \cdot T}\right)$

where $\tau_{0}$ is numerical coefficient and $\Delta E$ is an activation energy of the relaxation time. The relaxation time is a function of the distance a jumping electron must cover [32]. The distances between the individual particles are also random values from which a random distribution of the relaxation times values occur the thin films of $\mathrm{ZnO}$.

The comparative analysis of the dielectric permittivity and conductivity of the direct current indicates that the activation energy of the conductivity is higher than the activation energy of the permittivity. Indeed, we obtain $\Delta E_{\varepsilon}=3.23 \mathrm{meV}$ (Fig. 5) for the activation energy of the

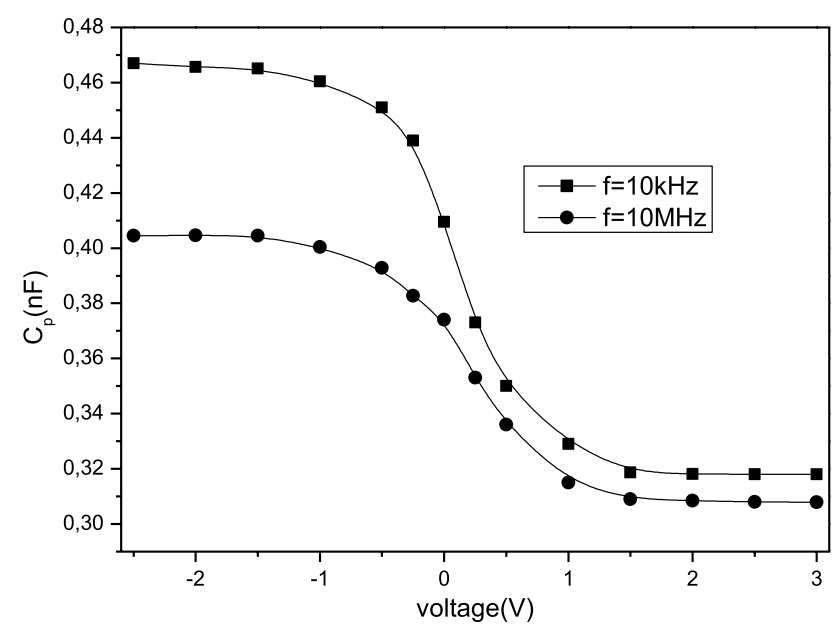

(a)

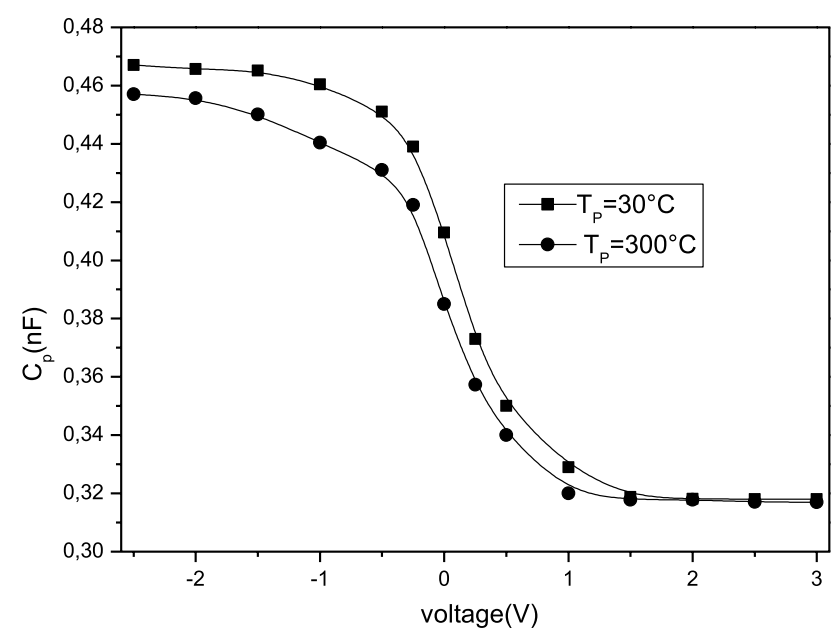

(b)

Fig. $7 C-V$ characteristics of MIS structure with $\mathrm{ZnO}$ films dependence of measurement frequency (a) temperature (b) 
dielectric permittivity and $\Delta E_{\sigma}=91.4 \mathrm{meV}$ (Fig. 6) for the activation energy of the low-frequency conductivity.

The measured $C-V$ characteristics of the studied $\mathrm{Pt} / \mathrm{ZnO} /$ $\mathrm{Ti} / \mathrm{Pt} / \mathrm{Si}$ structures are typical for a MIS structure. Figure 7 illustrates this assertion, where the $C-V$ characteristics of the MIS structure with a $\mathrm{ZnO}$ thin films deposited at $100^{\circ} \mathrm{C}$ at different frequencies and temperatures. The characteristics measured at $10 \mathrm{kHz}$ and $10 \mathrm{MHz}$ clearly revealed the accumulation, depletion and inversion regions in the $C-V$ plots of Fig. 7. The $10 \mathrm{MHz} C-V$ plot measured at room temperature was shifted toward more positive voltages compared with the ideal $C-V$ one. This shift has shown that there is fixed dielectric charge built-up in the $\mathrm{ZnO}$ thin films with a density of the order of $10^{17} \mathrm{~cm}^{-2}$, which has a weak tendency to increase with increasing temperature. The related defects were negatively charged, while the fixed oxide charge is always positive in a $\mathrm{ZnO}-\mathrm{Si}$ MIS structure. One may notice that with decreasing the measurement frequency, the capacitance values become larger in accumulation, depletion and inversion regions as presented in Fig. 7a. The $10 \mathrm{kHz} C-V$ characteristics showed a capacitance maximum in the depletion region, while toward the accumulation region capacitance increased little. The increase by almost one order of magnitude compared with the corresponding capacitance measured at $10 \mathrm{MHz}$ in the depletion region is evidence for the presence of traps at the $\mathrm{ZnO}-\mathrm{Si}$ interface and of deep levels in Si. The capacitance increase in the accumulation region signals the presence of deep levels in the bulk of $\mathrm{ZnO}$ thin films, which capture and emit charge carriers during the voltage sweeps. As illustrated in Fig. 7b, the $10 \mathrm{MHz} C-V$ characteristics measured at $30^{\circ} \mathrm{C}$ were shifted toward negative voltages relative to those measured at room temperature. This is an indication of the presence of positively charged traps at the $\mathrm{ZnO}-\mathrm{Si}$ interface, which contributes to the capacitance values moving the Fermi level toward the Si valence band edge during the temperature decrease from 300 to $30^{\circ} \mathrm{C}$. Therefore, the $\mathrm{ZnO}-\mathrm{Si}$ interface traps below the middle of the Si energy gap are donor like.

The electrical impedance at the input of the computed from the matrix (13) is $[33,34]$ structure:

$Z_{\mathrm{e}}=\frac{1}{j C_{0} \omega}+\frac{k^{2}}{C_{0} \omega} Z_{\mathrm{p}} \frac{2 Z_{\mathrm{p}}(\cos (\varphi)-1)+j\left(Z_{1}+Z_{2}\right) \sin (\varphi)}{Z\left(Z_{1}+Z_{2}\right) \cos (\varphi)+j\left(Z_{\mathrm{p}}^{2}+Z_{1} Z_{2}\right) \sin (\varphi)}$

where $Z_{\mathrm{p}}, Z_{1}, Z_{2}$, respectively, represent the acoustic impedances of the piezoelectric material, settings on the front and rear of the piezoelectric material. $k^{2}$ represents the electromechanical coupling coefficient

$C_{0}=\frac{\varepsilon_{\mathrm{r}} \cdot A}{d}$

$C_{\mathrm{p}}$ is the capacitance of the piezoelectric material and $\varphi$ is the constant.

To facilitate the study, first look at all the case of a free resonator. The model was developed to account for various losses related to the piezoelectric material $[34,35]$. We consider, for this, a thin piezoelectric layer, the two sides are metalized, and the electrical impedance is written in this case:

$Z_{\mathrm{e}}=\frac{1}{j C_{0} \omega}\left(1+K^{2} \frac{\tan (\varphi / 2)}{\varphi / 2}\right)=\frac{1}{j C_{0} \omega}\left(1+K^{2} \frac{\tan \left(\omega \mathrm{d} / 2 V_{\mathrm{p}}\right)}{\varphi \mathrm{d} / 2 V_{\mathrm{p}}}\right)$

Around the resonance, this resonator can be represented by the following equivalent electric circuit. $C_{0}$ is dielectric capacitance of the $\mathrm{ZnO}, L_{1}$ represents the inertia of the
Fig. 8 Electrical equivalent circuit of a piezoelectric resonator. a Model of Mason; $\mathbf{b}$ piezoelectric free

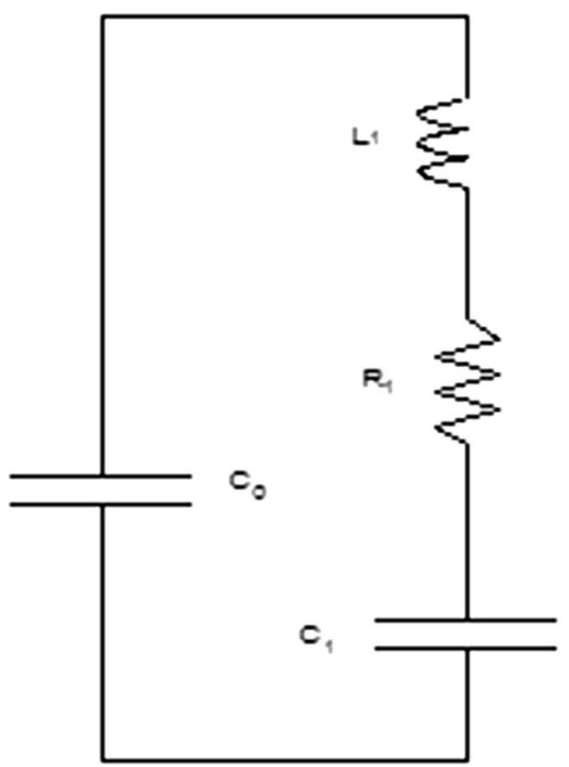

(a)

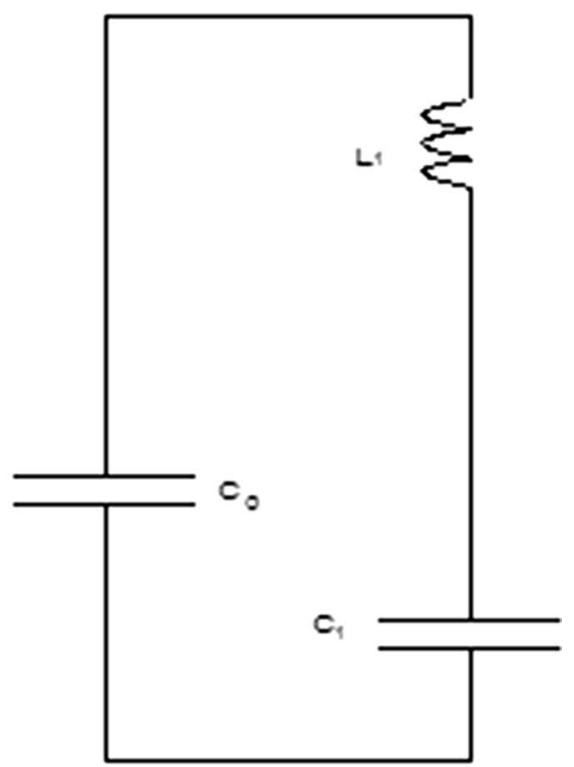

(b) 
circuit structure and the friction losses are $R$ and $C_{1}$ is stiffness of the system. The circuit in Fig. 8 is an ideal model to represent the physical characteristics of a free resonator. Indeed, when $\omega \rightarrow 0$, the parallel resonance frequency (frequency at which the maximum resistance is $R$ ) and series (frequency for which the conductance $G$ is a maximum) of the circuit may be written as follows:

$$
f_{\mathrm{S}}=\frac{1}{2 \pi \sqrt{L_{1} C_{1}}} \quad \text { et } \quad f_{\mathrm{p}}=\frac{1}{2 \pi \sqrt{L_{1} \frac{C_{1} C_{0}}{C_{1}+C_{0}}}}
$$

The IEEE standard [23] shows that the coupling coefficient $k^{2}$ is given by

$k^{2}=\frac{\varphi}{\tan \varphi} \quad$ with $\quad \varphi=\frac{\pi}{2} \frac{f_{\mathrm{S}}}{f_{\mathrm{p}}}$

The formula can be written in a more explicit form and approximate the error introduced by the approximation which is then less than $1 \%$ in the case where $k^{2}<0.2$, namely:

$k^{2}=\frac{\pi^{2}}{4} \frac{f_{\mathrm{S}}}{f_{\mathrm{p}}}\left(1-\frac{f_{\mathrm{S}}}{f_{\mathrm{p}}}\right)$

This means that one can calculate the coupling coefficient $k^{2}$ by simply raising the maximum of the real parts of the impedance and admittance, i.e., raise the maximum values of the impedance and admittance at the resonant frequency when the imaginary parts are zero. Using the relation (16), we plotted the theoretical evolution of the real and imaginary parts of the electric admittance of the resonator free, thick $5.7 \mu \mathrm{m}$, depending on the frequency. Since the modeling was done for the propagation of longitudinal waves of volume, we find a good coupling coefficient $k^{2}=7.8 \%, k=0.28$ for a pure longitudinal mode [33].

Figure 9 shows the variation of the real and imaginary parts of the electric admittance versus frequency. We observe a change in the electrical input admittance of the resonator and the appearance of the produced oscillations superimposed on the general envelope. These oscillations are due to the propagation medium. They reflect changes in real and imaginary parts of the electrical admittance measured. This means that the system reacts to the electrical load with a lag effect. Indeed, the shape of the curves shows that there is interference of waves propagating in the propagation medium which is the silicon substrate.

In support of our study, we also measured changes in the reflection coefficient as a function of frequency on a Smith

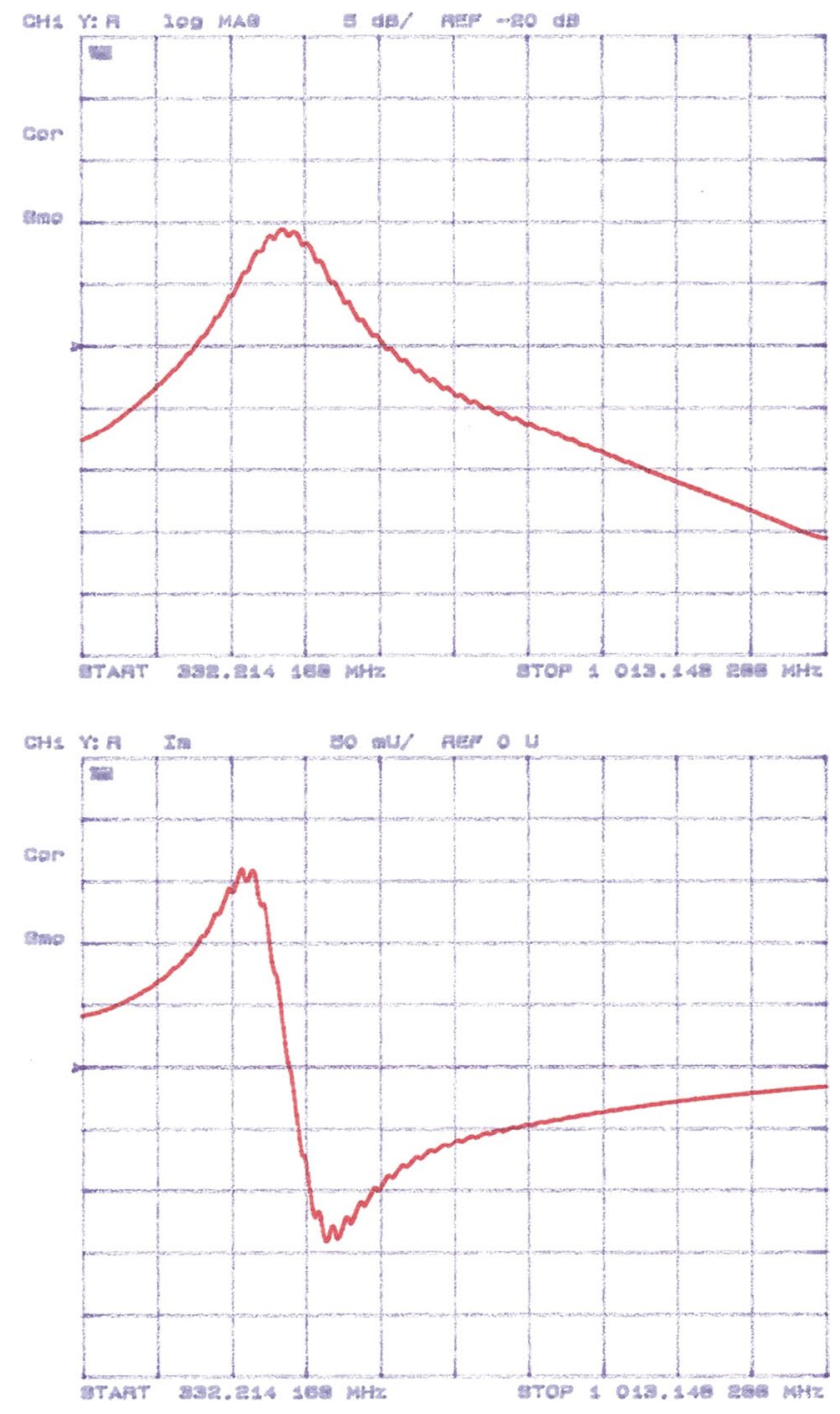

Fig. 9 RMS of admittance curves measured on the ZnO-based FBAR situated at the center of the wafer as function of frequency

chart. We observe the presence of the $\mathrm{ZnO}$ piezoelectric activity in Fig. 10.

To determine the coupling coefficient of our structure, we placed the fundamental resonance of the resonator, i.e., at frequencies where the real part of the admittance maximum and the minimum susceptance. We performed a piezoelectric resonator using the zinc oxide layer having a resonant frequency of the fundamental mode. The layer of zinc oxide and the delay line are, respectively, $3.8 \mu \mathrm{m}$ and $380 \mu \mathrm{m}$ thick. Figure 11 shows the evolution of the effective coupling coefficient $k_{\text {eff }}^{2}$ versus frequency. 
Fig. 10 Characteristic of $\mathrm{ZnO}$ based FBAR: Smith chart

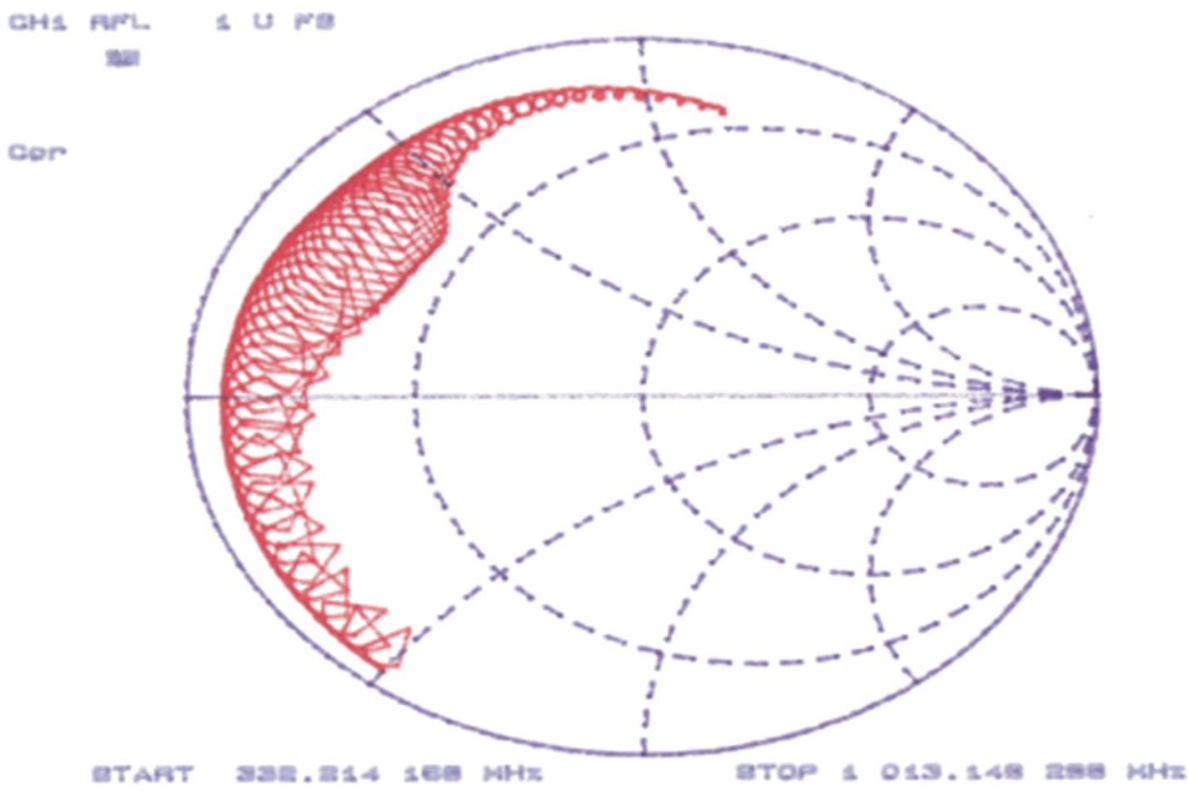

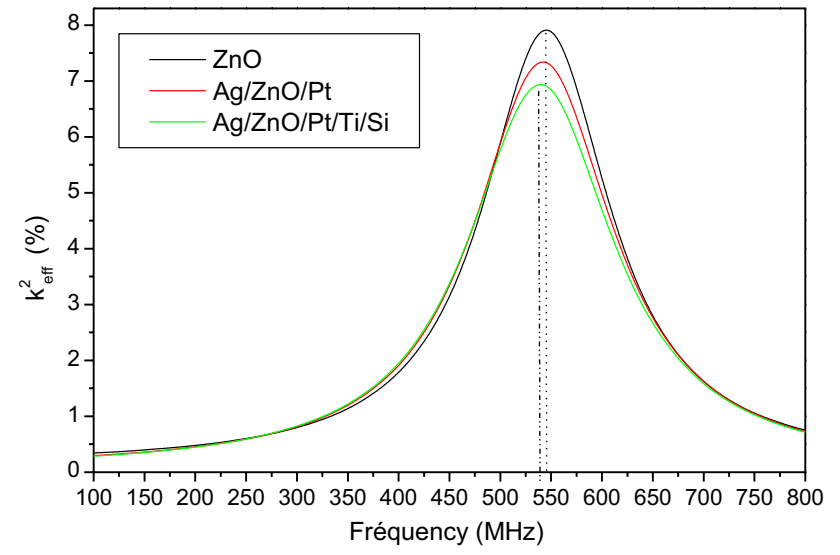

Fig. $11 k_{\text {eff }}^{2}$ function of the frequency of the $\mathrm{ZnO}$ resonator studied at $10 \mathrm{kHz}$ and $10 \mathrm{MHz}$ clearly revealed regions of accumulation, depletion and inversion in the plots. This offset showed that there was a fixed dielectric charge in the $\mathrm{ZnO}$ thin films with a density of the order of $10^{17} \mathrm{~cm}^{-2}$, hence a low tendency to increase with increasing temperature. The FBAR devices with the $\mathrm{ZnO}$ films exhibited a pronounced resonance peak centered at $537 \mathrm{MHz}$ with a $k^{2}$ coupling coefficient of $7 \%$.

Open Access This article is distributed under the terms of the Creative Commons Attribution 4.0 International License (http://creativeco mmons.org/licenses/by/4.0/), which permits unrestricted use, distribution, and reproduction in any medium, provided you give appropriate credit to the original author(s) and the source, provide a link to the Creative Commons license, and indicate if changes were made.

\section{Conclusion}

It has been observed that $\mathrm{AC}$ conductivity in $\mathrm{ZnO}$ films follows a $\sigma(\omega) \propto \omega^{s}$ dependence where $s \leq 1$ such behavior appears to indicate that hopping is the predominant conduction process. At low temperature and at high frequencies, observed values of $s$ approach those predicted by the literature that explains the behavior of AC conductivity of amorphous materials. The temperature and frequency dependences of the capacity and conductivity have been tested for a $\mathrm{ZnO}$ thin films prepared at a substrate temperature of $100{ }^{\circ} \mathrm{C}$. Frequency dependencies of capacity and conductivity indicate an appreciable overlap of the polaron hopping distortion which could occur (OLPT), thereby reducing the value of the polaron hopping energy. The measured current-voltage characteristics of the $\mathrm{Pt} / \mathrm{ZnO} / \mathrm{Ti} / \mathrm{Pt} / \mathrm{Si}$ structures

\section{References}

1. Pogrebnjak, A.D., Shpak, A.P., Azarenkov, N.A., Beresnev, V.M.: Structures and properties of hard and superhard nanocomposite coatings. Phys. Usp. 52, 29-54 (2009)

2. Pogrebnjak, A.D., Beresnev, V.M.: Nanocoatings nanosystems nanotechnologies, p. 147. Bentham Sci. Publ., Oak Park (2012)

3. Popok, V.N., Nuzhdin, V.I., Valeev, V.F., Stepanov, A.L.: Copper nanoparticles synthesized in polymers by ion implantation: surface morphology and optical properties. J. Mater. Res. 30(1), 86-92 (2015)

4. Himics, L., Toth, S., Veres, M., Toth, A., Koos, M.: Effective implantation of light emitting centers by plasma immersion ion implantation and focused ion beam methods into nanosized diamond. Appl. Surf. Sci. 328, 577-582 (2015)

5. Mahmood, F.S., Gould, R.D., Hassan, A.K., Salih, H.M.K.: DC properties of $\mathrm{ZnO}$ thin films prepared by RF magnetron sputtering. Thin Solid Films 270(1-2), 376-379 (1995) 
6. Salvadori, M.C., Teixeira, F.S., Sgubin, L.G., Cattani, M., Brown, I.G.: Surface modification by metal ion implantation forming metallic nanoparticles in an insulating matrix. Appl. Surf. Sci. 310, 158-163 (2014)

7. Kavitha, G., Arul, K.T., Babu, P.: Enhanced acetone gas sensing behavior of $n-\mathrm{ZnO} / p-\mathrm{NiO}$ nanostructures. J. Mater. Sci. Mater. Electron. 29, 6666-6671 (2018)

8. Poloju, M., Jayababu, N., Manikandan, E., Ramana Reddy, M.V.: Enhancement of the isopropanol gas sensing performance of $\mathrm{SnO} 2 / \mathrm{ZnO}$ core/shell nanocomposites. J. Mater. Chem. C 5, 2662-2668 (2017)

9. Lokesh, K., Kavitha, G., Manikandan, E., Mani, G.K., Kaviyarasu, K., Rayappan, J.B.B., Ladchumananandasivam, R., Aanand, J.S., Jayachandran, M., Maaza, M.: Effective ammonia detection using $n-\mathrm{ZnO} / p-\mathrm{NiO}$ heterostructured nanofibers. IEEE Sens. J. 16, 2477-2483 (2016)

10. Sathyaseelan, B., Manikandan, E., Sivakumar, K., Kennedy, J., Maaza, M.: Enhanced visible photoluminescent and structural properties of $\mathrm{ZnO} / \mathrm{KIT}-6$ nanoporous materials for white light emitting diode ( $w$-LED) application. J. Alloys Compd. 651, 479-482 (2015)

11. Manikandan, E., Kennedy, J., Kavitha, G., Kaviyarasu, K., Kamachi, U.: MudaliHybrid nanostructured thin-films by PLD for enhanced field emission performance for radiation micronano dosimetry applications. J. Alloys Compd. 647, 141-145 (2015)

12. Anusha Muthukumar, D., Arivuoli, E., Manikandan, M.Jayachandran: Enhanced violet photoemission of nanocrystalline fluorine doped zinc oxide (FZO) thin films. Opt. Mater. 47, 88-94 (2015)

13. Shah, A.H., Manikandan, E., Basheer Ahamed, M., Mir, Dilawar Ahmad, Mir, SajadAhmad: Antibacterial and blue shift investigations in sol-gel synthesized $\mathrm{Cr}_{x} \mathrm{Zn}_{1-x} \mathrm{O}$ nanostructures. J. Lumin. 145, 944-950 (2014)

14. Manikandan, E., Moodley, M.K., Sinha Ray, S., Panigrahi, B.K., Krishnan, R., Padhy, N., Nair, K.G.M., Tyagi, A.K.: Zinc oxide epitaxial thin film deposited over carbon on various substrate by pulsed laser deposition technique. J. Nanosci. Nanotechnol. 10, 5602-5611 (2010)

15. Manikandan, E., Murugan, V., Kavitha, G., Babu, P., Maaza, M.: Nanoflower rod wire-like structures of dual metal (Al and $\mathrm{Cr}$ ) doped $\mathrm{ZnO}$ thin films: structural, optical and electronic properties. Mater. Lett. 131, 225-228 (2014)

16. Manikandan, E., Kavitha, G., Kennedy, J.: Epitaxial zinc oxide, graphene oxide composite thin-films by laser technique for microRaman and enhanced field emission study. Ceram. Int. 40, 1606516070 (2014)

17. Manikandan, A., Manikandan, E., Meenatchi, B., Vadivel, S., Jaganathand, S.K., Ladchumananandasivam, R., Henini, M., Maaza, M., Aanand, J.S.: Rare earth element (REE) lanthanum doped zinc oxide ( $\mathrm{La}: \mathrm{ZnO})$ nanomaterials: synthesis structural optical and antibacterial studies. J. Alloys Compd. 723, 1155-1161 (2017)

18. Ondo-Ndong, R., Pascal-Delannoy, F., Boyer, A., Giani, A., Foucaran, A.: structural properties of zinc oxide thin films prepared by RF magnetron. Mater. Sci. Eng. B97, 68-73 (2003)

19. Komarov, F.F., Konstantinov, S.V., Pilko, V.V.: formation of nanostructured TiAlN, TiCrN and TiSiN coatings using reactive magnetron sputtering. J. Frict. Wear 35(3), 215-223 (2014)

20. Ondo-Ndong, R., Ferblantier, G., Pascal-Delannoy, F., Boyer, A., Foucaran, A.: Electrical properties of zinc oxide sputtered thin films. Microelectr. J. 34, 1087-1092 (2003)

21. Elliott, S.R.: A theory of AC conduction in chalcogenide glasses. Phil. Mag. 36(6), 1291-1304 (1977)

22. Pike, G.E.: AC conductivity of scandium oxide and a new hopping model for conductivity. Phys. Rev. B 6(4), 1572 (1972)

23. Elliott, S.R.: AC conductivity due to intimate pairs of charged defect centres. Solide Stat. Commun. 27, 749-751 (1978)

24. Lai, Li-Wen, Lee, Ching-Ting: Investigation of optical and electrical properties of $\mathrm{ZnO}$ thin films. Mater. Chem. Phys. 110(2-3), 393-396 (2008)

25. Jung, W.H.: AC conduction mechanisms of $\mathrm{Gd} 1 / 3 \mathrm{Sr} 2 / 3 \mathrm{FeO} 3$ ceramic. Phys. B Condens. Matter. 403, 636-638 (2008)

26. Sivakumar, N., Narayanasamy, A., Shinoda, K., Chinnasamy, C.N., Jeyadevan, B., Greneche, J.-M.: Electrical and magnetic properties of chemically derived nanocrystalline cobalt ferrite. J. Appl. Phys. 102(1), 013916-013923 (2007)

27. Long, A.R.: Frequency-dependent loss in amorphous semiconductors. Adv. Phys. 31, 553-637 (1982)

28. Frôhlich, H.: Theory of Dielectrics: Dielectric Constant and Dielectric Loss, 2nd edn. Oxford Science Publications, Oxford (1958)

29. Holstein, T.: Quantal occurrence-probability treatment of smallpolaron hopping. Phil. Mag. B 37(1), 49-62 (1978)

30. Psarras, G.C., Manolakaki, E., Tsangaris, G.M.: Dielectric dispersion and AC conductivity in-iron particles loaded-polymer composites. Compos. A Appl. Sci. Manuf. 34(12), 1187-1198 (2003)

31. Kudryashov, A.M., Mashin, A.I., Logunov, A.A., Chidichimo, G., De Filpo, G.: Frequency dependence of the electrical conductivity in Ag/PAN nanocomposites. Tech. Phys. 57(7), 965-970 (2012)

32. Zukowski, P.V., Partyka, J., Wegierek, P., Sidorenko, J.W., Szostak, J.A., Rodzik, A.: Dielectric properties of the semiconducting compounds $\mathrm{Cd}_{1-x} \mathrm{Fe}_{x} \mathrm{Te}$. Semiconductors 33(3), 276-278 (1999)

33. Royer D., Dieulesaint E., «Ondes élastiques dans les solides», Tome 2, ed. Masson (1996)

34. IEEE Standard on Piezoelectric IEEE/ANSI Std: 176 (1987)

35. Zhang, Y., Wang, Z., Cheeke, J.D.N.: Simulation of electromechanical coupling coefficient by modified modal frequency spectrum method including the electrode effect. Ultrasonics $\mathbf{3 8}$, 114-117 (2000) 